\title{
Las Innovaciones Educativas con TIC como generadoras de cambio en las prácticas pedagógicas de aula
}

Educational innovations with ICT as generators of change in classroom pedagogical practices.

Ulises Hernandez Pino ${ }^{1}$

Sandra Lorena Anaya Diaz ${ }^{2}$

Emigdio Andrés Lara Silva ${ }^{3}$

Mary Cristina Carrascal Reyes ${ }^{4}$

\section{RESUMEN}

En la Fase 1 del proyecto de investigación "Fortalecimiento y ampliación de cobertura de la formación postgradual en Innovaciones Educativas con TIC a través de estrategias de educación virtual pertinentes al contexto socio-cultural de la región", desarrollado por la Universidad del Cauca y en alianza con la Secretaria de Educación de Popayán y del Cauca, y la Corporación Cluster CreaTIC, se indagó sobre: el sentido que tienen las Innovaciones Educativas con TIC para los docentes en el sur-occidente colombiano; las tendencias mundiales sobre el uso de las nuevas tecnologías en educación a partir de los reportes del proyecto Horizon; y la revisión de los planes de estudio de 18 programas de postgrado en Colombia. La conclusión es que las Innovaciones Educativas con TIC, más que la incorporación de tecnología en la educación, busca generar cambios en el paradigma pedagógico a partir de las posibilidades que ofrecen estas nuevas tecnologías.

PALABRAS CLAVE: Innovaciones Educativas, TIC, Investigación-Acción Participativa.

\section{ABSTRACT}

In Phase 1 of the research project "Strengthening and expanding the coverage of postgraduate training in Educational Innovations with ICT through virtual education strategies in the socio-cultural context of the region", developed by the Universidad del Cauca and in alliance with the Secretariat of Education

\footnotetext{
Ingeniero en Electrónica y Telecomunicaciones y Candidato a Doctor en Ciencias de la Educación de la Universidad del Cauca. Director Académico de la Corporación ApropiACYT (Colombia). Correo electrónico: ulises@unicauca.edu.co

2 Sandra Lorena Anaya Diaz. Ingeniera en Electrónica y Telecomunicaciones y Especialista en Gerencia de Proyectos de la Universidad del Cauca. Directora Ejecutiva de la Corporación ApropiACYT (Colombia). Correo electrónico: sanaya@unicauca.edu.co

${ }^{3}$ Emigdio Andrés Lara Silva. Ingeniero en Electrónica y Telecomunicaciones de la Universidad del Cauca y Magíster en Ingeniería Eléctrica Área Telecomunicaciones. Jefe del Departamento de Telemática e investigador del Grupo de Ingeniería Telemática de la Universidad del Cauca (Colombia). Correo electrónico: alara@unicauca.edu.co

${ }^{4}$ Mary Cristina Carrascal Reyes. Ingeniera en Electrónica y Telecomunicaciones y Magíster en Ingeniería Telemática de la Universidad del Cauca. Docente del Departamento de Telemática y coordinadora del proyecto de investigación "Fortalecimiento y ampliación de cobertura de la formación postgradual en Innovaciones Educativas con TIC a través de estrategias de educación virtual pertinentes al contexto socio-cultural de la región” del Grupo de Ingeniería Telemática de la Universidad del Cauca (Colombia). Correo electrónico: mcarrasc@unicauca.edu.co
} 
of Popayán and of Cauca, and the Group of Creation of Companies, inquired about the meaning of Educational Innovations with ICT for teachers in south-western Colombia; global trends in the use of new technologies in education based on the Horizon project reports; and the revision of the study plans of 18 postgraduate programs in Colombia. The conclusion is that Educational Innovations with ICT, rather than the incorporation of technology in education, seeks to generate changes in the pedagogical paradigm based on the possibilities offered by these new technologies.

KEYWORDS: Educational Innovations, ICT, Participatory Action Research.

\section{INTRODUCCIÓN}

La aparición de las Tecnologías de la Información y las Comunicaciones (TIC) han planteado el reto de pensar en métodos alternativos de enseñanza y aprendizaje, si se quiere aprovechar estas herramientas de manera más efectiva en los procesos educativos. Las transformaciones potenciales de los procesos educativos debido a las TIC, es lo que ha motivado a los diferentes países del mundo a invertir en dotación de computadores y tabletas, acceso a Internet y formación de docentes en el sector educativo (Sunkel \& Trucco, 2010), buscando con ello las condiciones para pasar de una sociedad industrial a una sociedad del conocimiento (Castell, 2000; ).

La formación de docentes para aprovechar estas tecnologías es un componente esencial de esta política (Severin, 2010), pero planteada como cursos de cualificación o cursos cortos, los cuales resultan insuficientes. De otro lado, el diseño y desarrollo de programas formales de educación se ha realizado, tradicionalmente, a partir de las ideas planteadas por Franklin Bobbit en 1918: Definir la secuencia de temas o de experiencias de aprendizaje teniendo en cuenta el incremento paulatino en la complejidad, los tiempos y los recursos disponibles en la institución, para el cumplimiento de los objetivos de formación (Díaz Barriga, 2003, p. 5). Sin embargo, esta forma de planeación educativa no necesariamente permite la construcción de sentidos y las articulaciones de diferentes actores de la comunidad educativa, y por el contrario puede quedarse en una planeación de expertos que no contribuye con el desarrollo de programas de formación pertinente al contexto socio-cultural.

Por este motivo, el proyecto de investigación "Fortalecimiento y ampliación de cobertura de la formación postgradual en Innovaciones Educativas con TIC a través de estrategias de educación virtual pertinentes al contexto socio-cultural de la región" es desarrollado de forma colaborativa entre la Universidad del Cauca (sector académico), las
Secretarías de Educación de Popayán y el Cauca (sector gubernamental regional), y la Corporación Cluster CreaTIC (sector empresarial).

\section{Metodología}

Dado que el objetivo del proyecto es diseñar de forma participativa un programa virtual de postgrado en TIC para la Innovación Educativa, que considere la articulación con diferentes sectores, se consideró necesario realizar el estudio desde el enfoque de la Investigación-Acción Participativa (IAP). Como lo mencionan Kemmis y McTaggart, la IAP se diferencia de otras metodologías, en que el estudio se realiza de forma conjunta y la investigación se orienta hacia la acción colectiva (2005, p. 273).

La IAP plantea un modelo de reflexión y acción en espiral determinado por los participantes (Kemmis \& McTaggart, 2005), la adaptación de esta metodología para el proyecto, dio como resultado el planteamiento de las siguientes fases: 1) Identificación de necesidades y oportunidades de forma colectiva, sobre el uso de las TIC para impulsar innovaciones educativas; 2) Elaboración y realimentación de un documento con la definición de los ejes de formación del programa de posgrado, construido en consenso con los aliados del proyecto; 3) Montaje piloto de Cursos virtuales con metodologías que propendan por la articulación con el entorno.

En este texto se presentan los avances realizados en la Fase 1, en donde se han desarrollado 3 actividades: 1.1) Indagar de los significados que tienen las Innovaciones Educativas con TIC para los docentes de la región; 1.2) Identificar las tendencias mundiales del uso de las TIC para el desarrollo de innovaciones educativas; y 1.3) Examinar los planes de estudio de programas de postgrado del país relacionados con el tema de innovaciones educativas con TIC. 


\section{Resultados}

Respecto de la actividad 1.1, se desarrollaron 7 grupos focales que reunieron a 168 docentes de colegios públicos y privados, rurales y urbanos, de educación básica y educación superior, así como estudiantes de Escuelas Normales, en 3 departamentos de Colombia (Valle, Cauca y Nariño), con el propósito de conocer los significados que estos actores tienen sobre las Innovaciones Educativas con TIC. Esta focalización se realizó debido a que los docentes de educación básica y media, y docentes universitarios son la principal población objetivo del programa de postgrado.

Las respuestas dadas por los participantes en los grupos focales se analizaron desde la Teoría Fundamentada para su codificación y la definición de categorías (Strauss \& Corbin, 2002), en la cual se identificaron más de 300 códigos, los cuales se agruparon en 52 categorías. En una segunda etapa de análisis, se seleccionaron las 32 categorías más significativas, se agruparon y se buscó relaciones entre ellas para identificar la categoría central. La síntesis de este trabajo se encuentra en el mapa conceptual (ver Imagen 1).

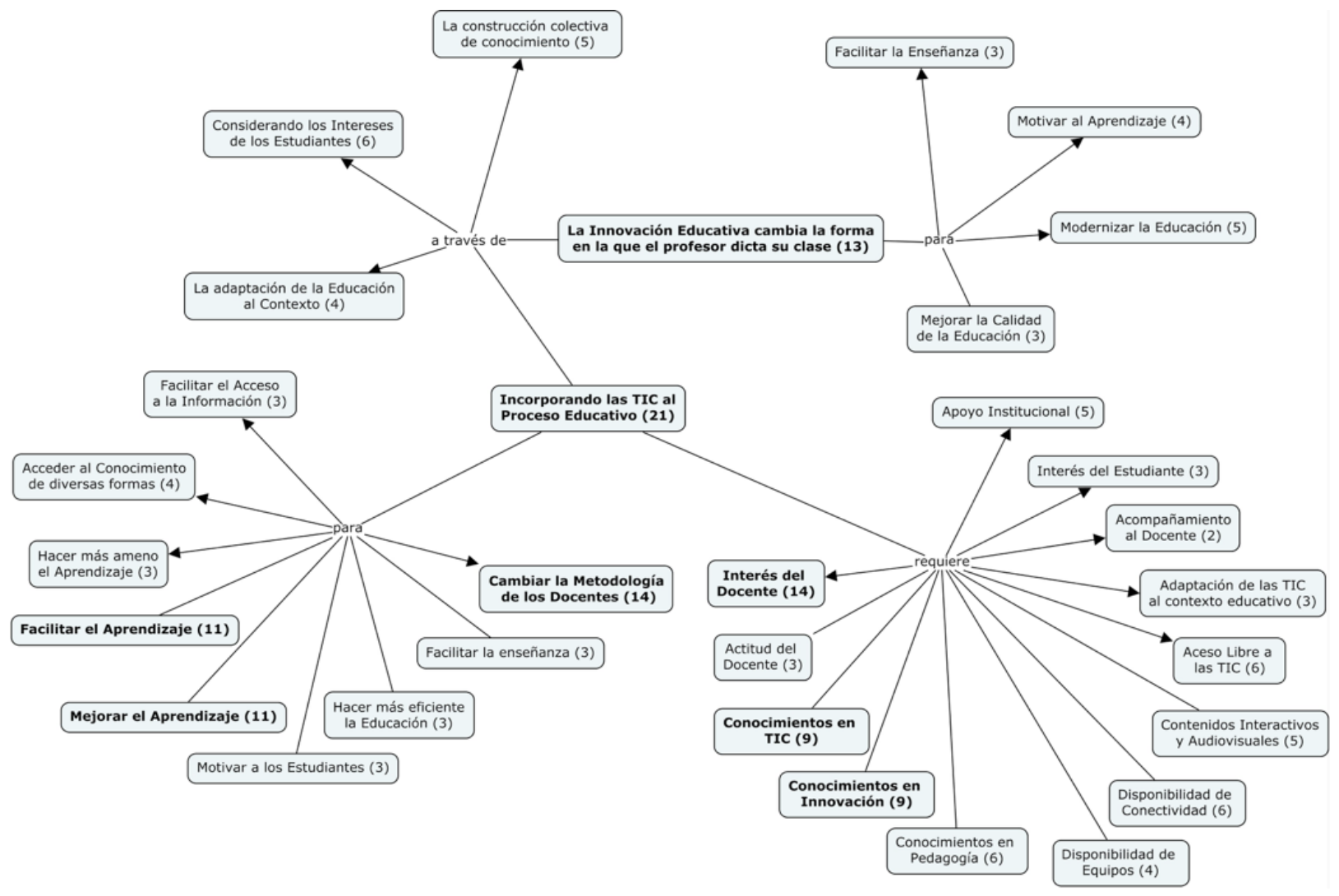

Imagen 2. Mapa conceptual con las categorías del significado que tiene las Innovaciones Educativas para los docentes en el sur-occidente colombiano

Para la actividad 1.2 se revisaron los reportes del proyecto Horizon del New Media Consortium (NMC), que desde el año 2004 estudia las tendencias de uso de las tecnologías para la enseñanza y el aprendizaje, a corto, mediano y largo plazo. De esta manera se buscó conocer el panorama del uso educativo de estas tecnologías a nivel mundial. En total se analizaron 34 documentos, de donde emergieron 8 categorías sobre las tecnologías planteadas en el periodo 2004 a 2018.

En cuanto a la actividad 1.3 se analizaron los planes de estudio de 18 programas de postgrados relacionados con innovaciones educativas con TIC, los cuales se buscaron a través del Sistema Nacional de Información de Educación Superior (SNIES) del Ministerio de Educación Nacional. De esta revisión se identificaron 4 categorías que agrupan las tendencias en la definición de cursos de formación a nivel de especializaciones y particularmente de maestrías. 


\section{Análisis}

A través de los grupos focales se identificó que los docentes asocian las Innovaciones Educativas con los cambios en la forma de dictar sus clases (ver categoría central en Imagen 1), y en este proceso la incorporación de las TIC juega un papel importante al facilitar los procesos de enseñanza, motivar el aprendizaje de los estudiantes, y ayudar a mejorar la calidad y pertinencia educativa.

Es de resaltar en este análisis, que las personas que participaron tienen claridad sobre la postura pedagógica desde la cual se pueden impulsar innovaciones educativas con las TIC, la cual se caracteriza por generar procesos que impulsen la construcción colectiva de conocimiento, a partir de los intereses de los estudiantes y teniendo en cuenta las particularidades del contexto sociocultura. Sin embargo, reconocen que requieren formación y acompañamiento para materializar estos cambios en el aula.

En cuanto a los reportes del proyecto Horizon, las diferentes tecnologías propuestas como tendencia en el periodo 2004 a 2018, se agruparon en las siguientes categorías: 1) Educación Virtual, 2) Realidad Virtual y Realidad Aumentada, 3) Analítica del Aprendizaje, 4) Gamificación, 5) Recursos Educativos Abiertos, 6) Contenidos creados por el usuario, 7) Pensamiento de Diseño, y 8) Colaboración Virtual. Este análisis ayuda a determinar las líneas de profundización, por lo menos desde el campo tecnológico, que deberían tenerse en cuenta un programa de postgrado sobre Innovaciones Educativas con TIC.

Finalmente, la revisión de los planes de estudio de postgrados en Colombia, permitió evidenciar cursos de formación comunes, los cuales se agruparon en 4 categorías: 1) Cambios pedagógicos y didácticos que generan las nuevas tecnologías; 2) Desarrollo acompañado de proyectos o experiencias educativas con TIC; 3) Manejo de plataformas virtuales de gestión del aprendizaje y 4) Producción digital y de recursos educativos.

\section{Conclusiones}

Con el desarrollo de la primera fase del proyecto de investigación, se hace claro que un programa de postgrado en Innovaciones Educativas con TIC debe hacer énfasis en el cambio de las posturas pedagógicas y en el desarrollo de propuestas didácticas, relacionadas con la construcción colectiva del conocimiento, el considerar los intereses de los estudiantes y la adaptación de la educación al contexto, a partir de las posibilidades que ofrece las nuevas tecnologías.

Si bien lo que se denomina nuevas tecnologías cambia de acuerdo con el momento y el lugar, unas tecnologías que son esenciales para apoyar los procesos educativos del presente y futuro: 1) Educación virtual y móvil, 2) Colaboración virtual y 3) Creación de contenidos digitales propios.

Con estos elementos, se busca crear un espacio de formación postgradual que no sólo responda a las demandas y expectativas de los actores educativos de la región, sino que también este acorde con las tendencias sobre el futuro de la tecnología en la educación.

\section{Agradecimientos}

El presente artículo hace parte del trabajo que se esta adelantando en el marco del proyecto "Fortalecimiento y ampliación de cobertura de la formación postgradual en Innovaciones Educativas con TIC a través de estrategias de educación virtual pertinentes al contexto socio-cultural de la región" liderado por el Grupo de Investigación en Ingeniería Telemática de la Universidad del Cauca y con la participación de la Corporación Cluster CreaTIC, la Secretaría de Educación del municipio de Popayán, la Secretaría de Educación del departamento del Cauca. Este proyecto es financiado por el programa InnovAcción Cauca.

\section{REFERENCIAS}

[1]. Castell, M. (2000). La sociedad red. Madrid: Alianza.

[2]. Barrera, F. \& Linden, L. (2009). The Use and Misuse of Computers in Education: Evidence from a Randomized Experiment in Colombia. Impact Evaluation Series, 29. Washington: World Bank.

[3]. Díaz Barriga, A. (2003). Currículum: Tensiones conceptuales y prácticas. Revista Electrónica de Investigación Educativa, 5 (2).

[4]. Kemmis, S. \& McTaggart, R. (2005). Participatory Action Research: Communicative Action and Public Sphere. In N.K. Denzin \& Y.S. Lincoln (Eds.). 
SAGE Handbook of Qualitative Research. California: Sage.

[5]. Rodríguez, C., Sánchez, F. \& Márquez, J. (2011). Impacto del Programa "Computadores para Educar" en la deserción estudiantil, el logro escolar y el ingreso a la educación superior. Documentos CEDE, 15. Bogotá: Universidad de los Andes.

[6]. Severin, E. (2010). Tecnologías de la Información y la Comunicación (TICs) en Educación: Marco conceptual e indicadores. Notas Técnicas, 6. Washington : BID.
[6]. Strauss, A. \& Corbin, J. (2002). Bases de la investigación cualitativa: Técnicas y procedimientos para desarrollar la teoría fundamentada. Medellín: Universidad de Antioquía

[7]. Sunkel, G. \& Trucco, D. (2010). Nuevas tecnologías de la información y la comunicación para la educación en América Latina: riesgos y oportunidades. Serie Políticas Sociales, 167. Santiago de Chile: CEPAL. 